\title{
HYPERSPECTRAL IMAGING FOR SKIN RECOGNITION AND BIOMETRICS
}

\author{
Cong Phuoc Huynh ${ }^{1}$ Antonio Robles-Kelly $y^{1,2}$ \\ ${ }^{1}$ School of Engineering, Australian National University, Canberra ACT 0200, Australia \\ ${ }^{2}$ National ICT Australia (NICTA)*, Locked Bag 8001, Canberra ACT 2601, Australia
}

\begin{abstract}
In this paper, we present a system for automatic spectral signature acquisition and recognition of skin from hyperspectral face imagery. In the acquisition step, hyperspectral cameras are used to capture multispectral or hyperspectral images of faces for skin recognition. The acquired signature may either be stored in a database for future testing or be used for purposes of identification. In the recognition step, the system accounts for variations in the illumination by recovering the light power spectrum in the scene and obtains the scene reflectance by normalising the input image radiance accordingly. Furthermore, incorporated into this system is a NonUniform Rational B-Spline (NURBS) compact descriptor of spectral reflectance for recognition purposes. We have employed this system as a profiling tool to classify a real-world multispectral face image database into separate ethnic groups.
\end{abstract}

Index Terms - multi-spectral imaging, hyper-spectral imaging, biometrics, reflectance, skin recognition, NURBS.

\section{INTRODUCTION}

For decades, hyperspectral imaging [1] has been an active area of research in remote sensing. However, until recently, hyperspectral images had only been available to a limited number of researchers. Furthermore, the few commercial hyperspectral imaging systems were mainly airborne ones which could not be used for ground-based applications. With the advent of commercial systems such as the Hyperspectral Image Intensified Camera System ${ }^{1}$ of OKSI, terrestrial spectral imaging is expected to become a sensing technology with ample applications in areas such as face recognition, biosecurity and surveillance.

In hyperspectral imaging, the imagery does not comprise brightness or colour values, but rather an "image cube". This image cube is constituted by a number of "slices", each of which represents a wavelength-resolved image. As a result,

* NICTA is funded by the Australian Government as represented by the Department of Broadband, Communications and the Digital Economy and the Australian Research Council through the ICT Centre of Excellence program.

${ }^{1}$ For more information see http://www.techexpo.com/WWW/optoknowledge/prodhiicsi.html every slice is a spatially resolved sample over the light spectrum. This integration of the spectral and spatial information produces a very information-rich representation of the scene and opens up the possibility of exploiting the interdependency of the spectral and spatial information to represent targets and subjects of interest in unique and novel ways.

Moreover, the spectral response of the material under study over a number of wavelengths permits the recovery of the reflectance spectra as a feature for material identification and recognition. Methods such as those presented in [2, 3, 4] often treat recognition as a pixel-based classification task, based upon individual spectral signatures. This is related to the chemistry of the object, where each signature is generally related to the object material. These methods hinge on the notion that different materials have different characteristic responses as a function of wavelengths, which can be used to provide descriptions of the target objects.

In this paper, we explore hyperspectral imaging as a profiling tool and present a system to classify subject faces into separate ethnic groups. We also explore the use of alternatives to raw spectra as a means to classification and recognition by presenting results for a number of reflectance descriptors. These are robust to changes in illumination, noise and surface geometry. The work presented here illustrates how these descriptors can be used for profiling and recognition tasks, such as those arising in biometrics or positive access technologies.

\section{IMAGE ACQUISITION \& PROCESSING SYSTEM}

In this section, we present a system for multispectral and hyperspectral imagery acquisition and processing for the purposes of skin recognition and ethnic group identification based upon spectral reflectance features. Here, we present both the acquisition equipment as well as an algorithm for material-intrinsic reflectance recovery and a compact spectral reflectance descriptor for recognition purposes.

The system presented here incorporates a spectral reflectance descriptor designed to be compact and efficient for classifying spectral data. At the current state-of-the-art, the research on spectral feature extraction from spectral images mainly focuses on the analysis of absorption bands and dimensionality reduction on the image cube. Unlike the previous work, the NURBS-based spectral descriptor allows an 
analytical representation of arbitrary free-form spectral data through the use of B-Spline basis functions, by which functional analysis on spectral curves, such as spectral derivative analysis, is possible. Furthermore, we also introduce a descriptor extraction algorithm that operates on the entire image cube simultaneously, rather than on a single spectrum [5].

\subsection{Apparatus}

Our apparatus includes a pair of OKSI Turnkey Hyperspectral Cameras for the purpose of ground-based image acquisition in the visible and near-infrared ranges. The cameras are equipped with Liquid Crystal Tunable Filters (LCTF) which allow spectral bands to be resolved up to $10 \mathrm{~nm}$ in both the visible (430-720 $\mathrm{nm})$ and near infrared (650-990 $\mathrm{nm}$ ) wavelength ranges. Incoming light is received by the front optics, and dispersed by the spectral filters, before passing through the relay optics to reach the imaging sensor. The CCD imaging sensor is capable of recording up to 12-bit monochromatic images with a spatial resolution of $1392 \times 1040$ pixels.

With this equipment, we collect a face image database of 51 human subjects, each captured under one of 10 directional light sources with varying directions and spectral power. The light sources are divided into two rows, one placed above and the other at the same height as the camera system. The main direction of the lights is adjusted to point towards the centre of the scene. To obtain the ground truth illuminant spectrum for each image, we measured the average radiance reflected from a white calibration target, i.e. a LabSphere Spectralon, illuminated by the light sources under consideration.

\subsection{Illumination Invariant Spectral Features}

In this section, we first present a method of recovering material reflectance that is robust to changes in illumination, surface shading and specularity. Later, we formulate a compact descriptor of spectral reflectance of materials for the purposes of material recognition.

\subsubsection{Illumination Spectrum Recovery}

Accurate information of illumination power spectra allows proper normalisation of the input image radiance to recover material-intrinsic reflectance. This information may be measured in laboratory conditions using a reference material. However, in certain circumstances such as outdoor settings with varying natural sunlight power and direction, this calibration process becomes time-consuming and laborious. We have introduced a method of predicting the illumination power spectrum, up to a scaling constant, from a single hyperspectral image [6]. The method also enables the recovery of material-intrinsic reflectance which is invariant to illumination, surface shading and specularity.

\subsubsection{NURBS-based Spectral Reflectance Descriptor}

Having obtained the spectral reflectance as described in [6], we proceed by formulating a compact representation of reflectance spectra using B-Spline basis functions. By treating each reflectance spectrum as a set of 2-D points whose coordinates are corresponding pairs of wavelengths and reflectance, we aim to interpolate a B-spline curve to each spectrum and perform knot removal to minimise the number of knots and control points.

Suppose that we are given a collection of wavelengthindexed reflectance spectra as input, in the form of $\mathbf{R}_{v}=$ $\left[R_{v, 1}, R_{v, 2}, \ldots, R_{v, l}\right]^{T}$, where $v$ denotes the spectrum index (or pixel index in case of hyperspectral images) and $R_{v, k}$ is the reflectance of the spectrum $v$ sampled at the discrete wavelength $\lambda_{k}, k=1,2, \ldots, l$. We aim to interpolate a set of B-spline curves of the same degree $p$, with a common knot vector and the same basis functions to the input reflectance spectra. For the spectrum index $v$, suppose that the corresponding curve $\mathcal{C}_{v}$ is defined by $n+1$ control points $P_{v, i}=\left(x_{i}, y_{v, i}\right), i=0, \ldots, n$, and a common knot vector $U$ for all the given spectra, where $U=\left\{u_{0}, \ldots, u_{m}\right\}$, $m=n+p+1$. The wavelength and reflectance coordinates on this curve are defined as functions of an independent parameter $t$

$$
\begin{aligned}
\lambda(t) & =\sum_{i=0}^{n} N_{i, p}(t) x_{i} \\
\mathcal{R}_{v}(t) & =\sum_{i=0}^{n} N_{i, p}(t) y_{v, i}
\end{aligned}
$$

In Equations 1 and 2, $N_{i, p}(t)$ is the $i^{\text {th }}$ B-spline basis function of degree $p$, as defined in [7]. To provide a common basis for recognition and comparison between different spectra, we enforce a common set of basis functions, knot vector $U$ and control point coordinates $x_{i}$ in the wavelength dimension for all the given spectra. In contrast, the reflectance interpolation function $\mathcal{R}_{v}(t)$ and its associated control point coordinates $y_{v, i}$ characterise the input spectral signatures.

In pattern recognition, a descriptor is desired to contain characteristic information that yields high discriminative power. Hence, we derive a target function to optimise the choice of the interpolating B-spline curves for a given set of reflectance spectra. Here, the cost function aims to minimise both the reconstruction error of the interpolated curves with respect to the input spectra and the descriptor length.

Suppose that the parameter $t_{k}$ corresponds to the $k^{t h}$ wavelength, i.e. $\lambda_{k}=\lambda\left(t_{k}\right) \forall k$. Then the interpolation cost using the B-spline curves above through the set of reflectance spectra $\left\{\mathbf{R}_{v} \mid \mathbf{R}_{v}=\left[R_{v, 1}, R_{v, 2}, \ldots, R_{v, l}\right]^{T}\right\}$ is given by

$$
K=\alpha \frac{1}{N} \sum_{v} \sum_{k=1}^{l}\left(\mathcal{R}_{v}\left(t_{k}\right)-R_{v, k}\right)^{2}+(1-\alpha)|U|
$$


where $N$ is the number of spectral signatures, $|$.$| denotes the$ length of the vector argument. The choice of the balance factor $\alpha \in[0,1]$ governs the trade-off between the reconstruction error and the descriptor length.

Next, we turn our attention to the task of minimising the cost function in Equation 3. The algorithm departs from an initial interpolation of the sampled reflectance spectra so as to arrive at a set of curves that minimises the cost function through a knot removal algorithm. To initialise the B-Spline curves, we apply the curve interpolation algorithm in [7], which employs the centripetal method of Lee [8] to recover the curve parameter values for every sampled reflectance spectrum.

With these initial curves at hand, we proceed to remove knots sequentially so as to maximise the total cost reduction. The algorithm is a two-pass one. In the first pass, removable knots are identified. Once they are determined as removable, our algorithm computes the potential cost reduction for their removal. Then, the knot that yields the maximum cost reduction is selected for removal. In the second pass, we employ Tiller's algorithm [9] to remove the selected knot and its corresponding control point, while recomputing the other control points in the local support to best fit the given spectral reflectance samples. This process iterates until either a target number of knots has been achieved or the reconstruction error exceeds a preset threshold.

Note that this iterative process can be interleaved with a resampling operation in the parameter domain. The resampling operation allows further knots to be removed by reducing the number of curve sections fitted to the spectra without changing the distribution of the control points. Hence, the knot removal method described above can be applied recursively on the resampled data points in the parameter domain.

\section{ETHNIC GROUP PROFILING}

In this section, we illustrate the utility of the system described in Section 2.2.2 for the task of ethnicity identification from skin reflectance. First we compact the input reflectance image cube along the spectral dimension to obtain a third-degree BSpline curve with 18 control points and 22 knots. For recognition purposes, we reconstruct a reflectance vector from these optimal knots and control points, according to Equations 1 and 2.

We also consider alternative methods of feature extraction from reflectance spectra. The first of these consists of 15 principal components yielded by Linear Discriminant Analysis (LDA) on a vector made of the coefficients, means and standard deviations of a mixture of 6 Gaussian components fitted to the given reflectance spectra [10]. We also compare the performance of the NURBS descriptor with the raw reflectance spectra consisting of 30 spectral bands, and their 15 principal LDA components extracted from the raw spectra.

We perform the experiment on the visible face images from the database described in Section 2.1. Here, we obtain reflectance images by normalising the raw multispectral images with the groundtruth illumination power spectrum. Note that, as an alternative, the illumination power spectrum may also be estimated from single multispectral radiance images, as discussed in [6].

The dataset involved in this illustration consists of three different ethnic groups, including 22 Caucasian, 8 Indian and 18 Oriental subjects. The training data includes spectral signatures of 5 rectangular skin regions, with an average size of $25 \times 17$, from the frontally-illuminated images of two typical representatives of each ethnic group. A Support Vector Machines (SVM) classifier with a first-degree polynomial kernel is then trained on the spectral features, with its parameters tuned through a 5 -fold cross validation procedure. During the

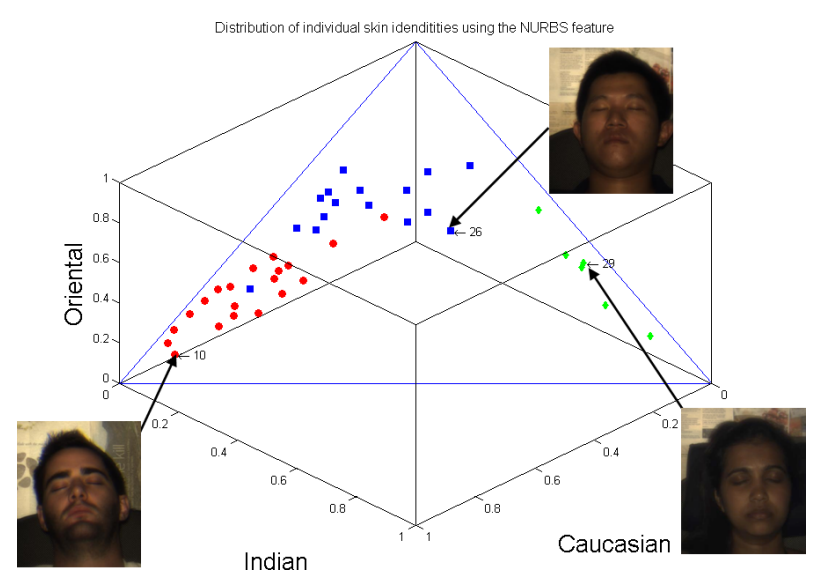

(a) NURBS feature

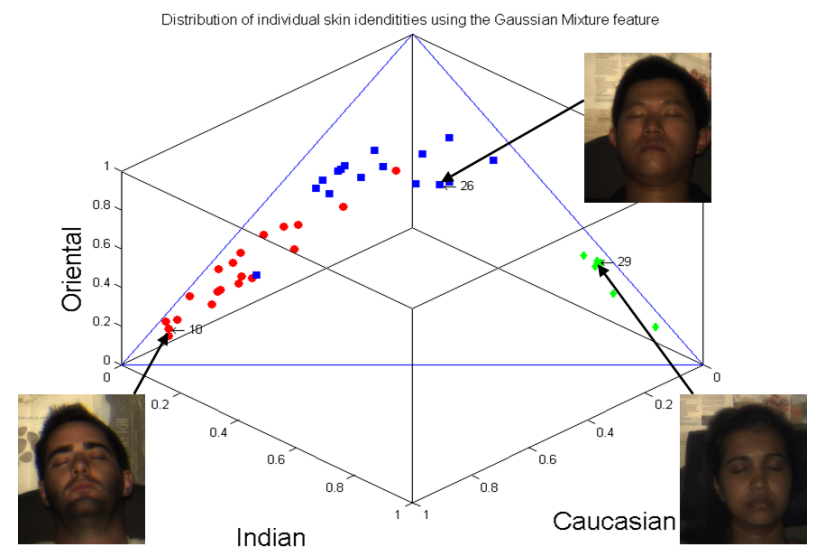

(b) Gaussian Mixture feature

Fig. 1. Visualisation of the association of individuals with three ethnic groups in a 3D coordinate system with the three axes corresponding to the groups. Sample face images of the ethnic groups are shown in pseudo RGB colours. (a) a plot of the class association probabilities yielded by the third-degree NURBS feature with 22 control points and 18 knots; (b) a plot of the class association probabilities yielded by the 15 principal LDA components of the Gaussian Mixture feature with 6 components. 


\begin{tabular}{|c|c|c|c|c|c|c|c|c|c|c|c|c|}
\hline & \multicolumn{3}{|c|}{ NURBS } & \multicolumn{3}{|c|}{ Gaussian Mixture } & \multicolumn{3}{|c|}{ Raw Spectra } & \multicolumn{3}{|c|}{ Raw Spectra + LDA } \\
\hline $\begin{array}{ll} & \text { Classified } \\
\text { Actual } & \end{array}$ & $\mathrm{C}$ & I & $\mathrm{O}$ & $\mathrm{C}$ & I & $\mathrm{O}$ & $\mathrm{C}$ & I & $\mathrm{O}$ & $\mathrm{C}$ & I & $\mathrm{O}$ \\
\hline Caucasian (C) & 95 & 0 & 5 & 90 & 0 & 10 & 90 & 0 & 10 & 90 & 0 & 10 \\
\hline Indian (I) & 0 & 83.33 & 16.66 & 0 & 100 & 0 & 0 & 83.33 & 16.66 & 0 & 100 & 0 \\
\hline Oriental $(\mathrm{O})$ & 12.5 & 0 & 87.5 & 6.25 & 0 & 93.75 & 6.25 & 0 & 93.75 & 6.25 & 0 & 93.75 \\
\hline
\end{tabular}

Table 1. The percentage of test subjects classified into the Caucasian (C), Indian (I) and Oriental (O) ethnic groups, using different spectral descriptors. Columns 2-4 result from the use of the NURBS-based descriptor with 18 control points. Columns $5-7$ are yielded using 15 LDA components of a Gaussian Mixture descriptor with 6 components. Columns 8-10 are yielded using 30 spectral bands of raw reflectance. Columns 11-13 are yielded using 15 principal LDA components of the raw spectra.

test phase, the trained SVM classifier assigns an ethnic group association probability to each skin pixel in the test images. Note that skin regions may be segmented from the test images in a manner similar to that in [6]. The overall ethnic identity associated with the test subjects is determined by the majority voting of the ethnic group association across their skin pixels.

In Figure 1, we visualise the ethnic group association probabilities of the test subjects as points in a 3-D coordinate system, with the three axes representing the ethnic groups. The ground-truth ethnic groups of the test subjects are colourcoded, as red, green and blue for Caucasian, Indian and Oriental, respectively. In both plots, the Indian skin cluster is well-separated from the other groups. This is expected as the Indian skin reflectance spectra from our database have flatter slopes and less absorption bands than the other two groups. The Caucasian and Oriental groups are fairly separable from each other with a few examples confounded by image saturation. It is also noted that each ethnic group is correctly located in a proximity of an extreme point corresponding to the pure skin example of the group.

In Table 1, we report the percentage of individuals classified into the ethnic groups as a confusion matrix between the groups. While the NURBS-based descriptor outperforms the others in recognising Caucasian skin, the application of LDA to the Gaussian Mixture feature and raw spectra yields a better recognition rate for the other groups. Although there is a slight difference in classification accuracy between the above spectral reflectance features, the plots in Figure 1 show similar distributions of invididual skin reflectance identity. This can be explained by the fact that the difference in classification accuracy could be attributed to the decision boundary determined by the SVM classifier, rather than the difference between spectral descriptors.

\section{CONCLUSION}

In this paper, we have provided the design and implementation of a multispectral imaging system for skin biometric applications. The first component of our system comprises an apparatus for acquiring skin reflectance samples from multispectral or hyperspectral images. The second component of the system is a collection of algorithms to recover material reflectance robust to illumination changes and to represent spectral reflectance of materials, including skin, in an efficient and compact form for classification purposes. We have also demonstrated the utility of this system as an ethnic group profiling tool.

\section{REFERENCES}

[1] Chein-I Chang, Hyperspectral Imaging: Techniques for Spectral Detection and Classification, Springer, 2006.

[2] D. Landgrebe, "Hyperspectral Image Data Analysis," IEEE Signal Process. Mag., vol. 19, pp. 17-28, 2002.

[3] L. Jimenez and D. Landgrebe, "Hyperspectral Data Analysis and Feature Reduction via Projection Pursuit," IEEE Transaction on Geoscience and Remote Sensing, vol. 37, no. 6, pp. 2653-2667, 1999.

[4] M. Dundar and D. Landgrebe, "Toward an Optimal Supervised Classifier for the Analysis of Hyperspectral Data," IEEE Transaction on Geoscience and Remote Sensing, vol. 42, no. 1, pp. 271-277, 2004.

[5] Cong Phuoc Huynh and Antonio Robles-Kelly, "A NURBSbased Spectral Reflectance Descriptor with Applications in Computer Vision and Pattern Recognition," IEEE Conference on Computer Vision and Pattern Recognition, pp. 1-8, 2008.

[6] Cong Phuoc Huynh and Antonio Robles-Kelly, "A Solution of the Dichromatic Model for Multispectral Photometric Invariance," International Journal of Computer Vision, vol. 90, no. 1, pp. 1-27, 2010.

[7] Les Piegl and Wayne Tiller, The NURBS book, SpringerVerlag, London, UK, 1995.

[8] E. T. Y. Lee, "Choosing nodes in parametric curve interpolation," Computer-Aided Design, vol. 21, no. 6, pp. 363-370, 1989.

[9] Wayne Tiller, "Knot-removal algorithm for NURBS curves and surfaces," Computer-Aided Design, vol. 24, no. 8, pp. 445, 1992.

[10] E. Angelopoulou, R. Molana, and K. Daniilidis, "Multispectral skin color modeling," in IEEE Conf. on Computer Vision and Pattern Recognition, 2001, pp. II:635-642. 